\title{
Fully engineered homoepitaxial zinc oxide nanopillar array for near-surface light wave manipulation
}

\author{
J. Volk, ${ }^{1,2, a)}$ A. Håkansson, ${ }^{2,3}$ H. T. Miyazaki, ${ }^{2}$ T. Nagata $^{2}{ }^{2}$ J. Shimizu, ${ }^{2}$ and T. Chikyow ${ }^{2}$ \\ ${ }^{1}$ Research Institute for Technical Physics and Materials Science, H-1121 Budapest, \\ Konkoly Thege Miklós út 29-33, Hungary \\ ${ }^{2}$ National Institute for Materials Science, 1-1 Namiki, Tsukuba, Ibaraki 305-0044, Japan \\ ${ }^{3}$ DAS Photonics S.L., Ciudad Politécnica de la Innovacion, Camino de Vera s/n, 46022 Valencia, Spain
}

(Received 11 March 2008; accepted 19 April 2008; published online 9 May 2008)

\begin{abstract}
We report accurate light wave manipulation by means of an inverse designed homoepitaxial $\mathrm{ZnO}$ nanopillar array. Our proof-of-concept structure was optimized for focusing a near-surface light beam which propagates in a free-space parallel to the metal top surface. The on-purpose positioned and perpendicularly aligned vertical $\mathrm{ZnO}$ nanopillars were fabricated by homoepitaxial chemical growth technique. The obtained focal distance of $28 \mu \mathrm{m}$ as well as the light intensity distribution pattern was verified by three-dimensional finite-difference time-domain method. The demonstrated approach can provide inter- and intrachip optical connections in the next generation $\mathrm{ZnO}$ nanowire-based integrated photonic devices. (C) 2008 American Institute of Physics.
\end{abstract}

[DOI: $10.1063 / 1.2924282]$

Zinc oxide as a wide band gap semiconductor $(3.37 \mathrm{eV})$ that has a large exciton binding energy $(\sim 60 \mathrm{meV})$ is predestinated to be a key material of the future nanophotonic devices operating in the visible and in the near ultraviolet wavelength region. The intense interest is partly due to the unique bulk properties of $\mathrm{ZnO}^{1}$ and partly because of its ability to form stable nanostructures. ${ }^{2}$ In the past decade, several optical phenomena and functions, such as blue-UV light emission, ${ }^{3}$ UV lasing, ${ }^{4}$ strong second harmonic generation, ${ }^{5}$ and optical switching, ${ }^{6}$ were demonstrated either on individual $\mathrm{ZnO}$ nanowires (NWs) or on vertical NW arrays. Moreover, by the latest development in $p$-type NW doping, ${ }^{7}$ the most crucial obstacle of $\mathrm{NW}$ based $\mathrm{ZnO}$ devices can be overcome as well. This enormous progress makes us envision the emergence of all- $\mathrm{ZnO}$ integrated devices; however, the essential inter- and intrachip connections have been still missing.

The efficient device integration always make a great demand on the technological compatibility. In ideal case, the constituent optical components are fabricated in the same parallel process. Here, we propose an innovative concept in which a vertical $\mathrm{ZnO}$ nanopillar array is used for the manipulation of the light wave propagating parallel to the surface in free space. The array is very similar to the above listed active counterparts, therefore, promising easy integration. The selected solution is based on the scattering optical element (SOE) concept $^{8}$ which can be regarded as the threedimensional (3D) extension of the diffractive optical elements (DOE). However, instead of using a single layer of phase-controlling component, SOEs are based on multiple scattering by a few layers of individual scatterers. The positions of the scatterers are optimized by a powerful combination of two-dimensional (2D) multiple scattering theory and genetic algorithm, ensuring a fast and effective optimization. According to the simulations, the inverse designed SOE structures can fulfill different functions such as light focusing and incoupling of an external source, ${ }^{9}$ directional light

\footnotetext{
${ }^{a)}$ Electronic mail: volk@mfa.kfki.hu.
}

emission, ${ }^{10}$ wavelength demultiplexing, ${ }^{8}$ etc. Although proof-of-concept structures have already been demonstrated, they either operate in the microwave region ${ }^{11}$ or they are optimized for acoustic waves. ${ }^{12} \mathrm{SOE}$ structures operating in the optical region still have been missing.

In order to demonstrate the capabilities of the concept, here we present a nanopillar lens which focuses the light of an external monochromatic source with $\lambda=633 \mathrm{~nm}$ (Fig. 1). For an easy demonstration of the optical function, a focal distance of $30 \mu \mathrm{m}$ was selected; however, there would be no physical limitation of a significantly lower value. The optimization process was applied on a narrow rectangular region having a triangular lattice of $n \times m=71 \times 3$, where each site can be either an occupied or a vacant position for infinitely long circular cylinders. It is possible to remove this limitation on the design freedom and freely place the scatterers in the plane, ${ }^{11}$ although an increase in the performance might be expected, the array is normally more difficult to realize. The final arrangement, which is one of the $2^{(n+1) / 2 m}$ $\approx 3.24 \times 10^{32}$ possible $z$-axis symmetric configurations, was selected by maximizing the intensity gain factor in the focal point. The optimal configuration, with a gain factor of

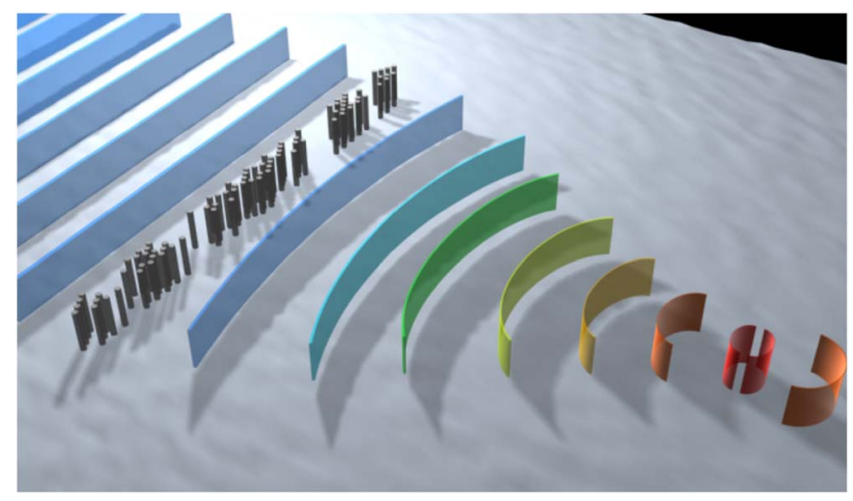

FIG. 1. (Color online) A schematic cartoon of the optical lens system. The sheets mark the wave front as the light propagates from left to the right through the SOE nanopillar device. The focal point is set to $30 \mu \mathrm{m}$ behind the front edge of the array. 

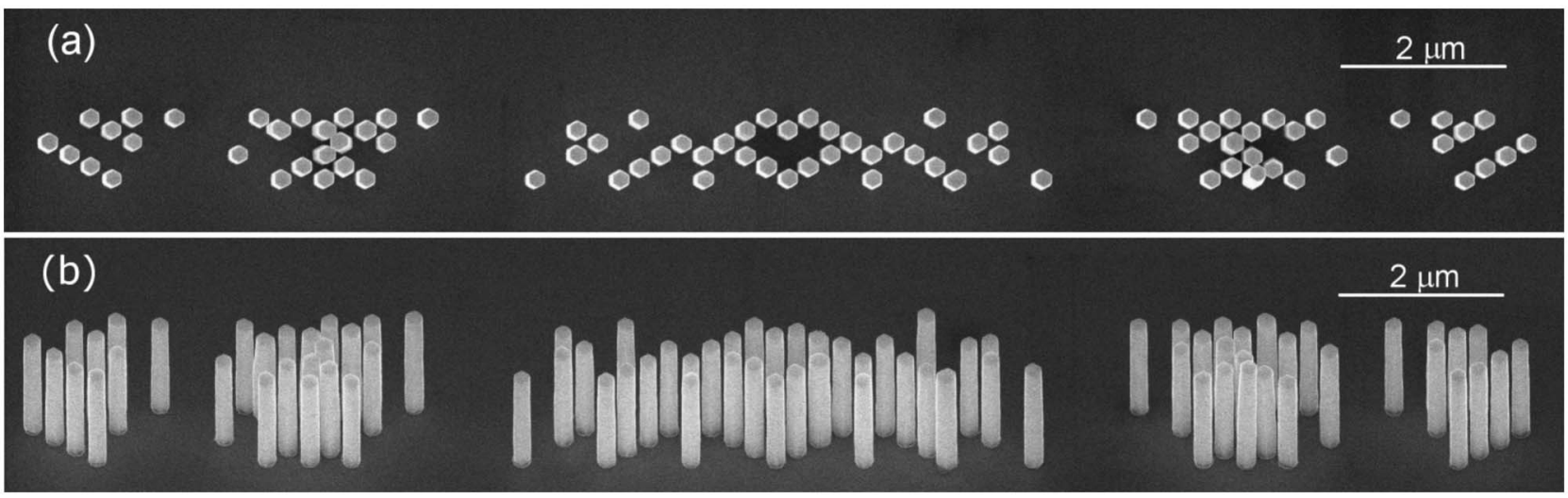

FIG. 2. Top (a) and perspective view (b) scanning electron microscopic (SEM) images taken on the homoepitaxial ZnO nanopillar array. The highly uniform 2.1- $\mu \mathrm{m}$-long prism-shaped vertical nanocrytals are collectively aligned according to the crystal orientation of the wurtzite-type ZnO substrate.

$G=11.6$, was found after 6800 function evaluation, equalling approx. $3 \mathrm{~h} \mathrm{CPU} \mathrm{time} \mathrm{on} \mathrm{a} \mathrm{standard} \mathrm{Pentium} \mathrm{IV} \mathrm{processor}$ and using only a few megabyte random access memory.

In the applied 2D optimization method, the planar light wave is modulated by infinitely long freestanding scatterers; however, experimentally obtained structures are finitely long nanopillars which are standing on the surface. Hence, a 3D finite-difference time-domain (FDTD) method was used which unambiguously confirmed that the predesigned optical function is attained by such a realistic geometry.

The next practical question is how can we fabricate a perpendicularly aligned $\mathrm{ZnO}$ nanopillar array in an engineered arrangement. On purpose positioned arrays were reported only recently by Kim et al. ${ }^{13}$ and by Cui et al. ${ }^{14}$ Our synthesis method is partly similar to them, we also use e-beam lithography for generating the designed pattern and soft chemical nanocrystal synthesis. ${ }^{15}$ However, we propose $\mathrm{ZnO}$ single crystal as a substrate material and a homoepitaxial nanopillar growth, enabling significantly enhanced regularity and uniformity throughout the array.

The fabrication process began with the deposition of a high quality $\mathrm{Pt}$ thin film on the $\mathrm{Zn}$-terminated (0001) face of a single crystalline $\mathrm{ZnO}$ substrate ${ }^{16}$ to ensure the low loss propagation for transverse electric (TE)-polarized light. The thickness $(39.3 \mathrm{~nm})$ and complex refractive index (2.03 $+4.00 i$ at $\lambda=633 \mathrm{~nm}$ ) of the thin Pt film were determined precisely by a combined method of $\mathrm{x}$-ray reflectivity and spectroscopic ellipsometry. The desired pattern was drawn at first in a poly(methyl methocrylate) (PMMA) layer by e-beam lithography which was transferred then into the $\mathrm{Pt}$ layer by $\mathrm{Ar}^{+}$ion beam etching. Here, we carefully optimized the etching time to form Pt free but only slightly damaged $\mathrm{ZnO}$ nucleation centers in the circles on the selected lattice sites. After the removal of the residual PMMA layer, the nanopillars were synthesized at $85^{\circ} \mathrm{C}$ in a sealed glass container filled with an equimolar $(0.004 \mathrm{M})$ aqueous solution of zinc nitrate hexahydrate $\left[\mathrm{Zn}\left(\mathrm{NO}_{3}\right)_{2} \cdot 6 \mathrm{H}_{2} \mathrm{O}\right]$ and hexamethylenetetramine $\left(\mathrm{C}_{6} \mathrm{H}_{12} \mathrm{~N}_{4}\right)$.

The obtained defect-free array consists of uniform, $2.1 \mu \mathrm{m}$ long nanopillars standing perpendicular on the surface (Fig. 2). Although the patterned Pt film contains circular bores the nanopillars show hexagonal cross sectional shapes which are collectively aligned according to the inherent crystal orientation of the wurtzite-type $\mathrm{ZnO}$ substrate. Multiple scattering simulations did not show any notable difference between rods with circular and hexagonal cross sections at Author complimentary copy. Redistribution subject to AIP the working wavelength, making our design valid. The homoepitaxial pillars are slightly tapered: the distance between the nonpolar [10-10] side planes changes between 190 and $230 \mathrm{~nm}$ when going from the bottom to the tip. This feature can be ascribed to the fact that the growing rate ratio between polar top [0002] and nonpolar side planes [10-10] is large but not infinite.

In the test measurement, the effect of the SOE structure on the in-plane near-surface light propagation was investigated by means of a far field quasi-plane-wave source. However, when setting a parallel incident direction for a collimated laser beam and trying to attain a grazing propagation, due to the Lloyd's mirror effect, ${ }^{17}$ the light intensity inevitably vanishes near to the surface. On the other hand, by carefully adjusting the grazing incidence angle so that the first constructive interference band sufficiently covers the SOE structure with a finite height, a plane wave incident from a far field can mimic the in-plane near-surface light propagation induced, e.g., by an emitter on the same $\mathrm{ZnO}$ optical chip. It was found that a grazing angle of $3.7^{\circ}$ fulfills this requirement for the present structure when using a TEpolarized collimated laser beam of $\lambda=633 \mathrm{~nm}$. The scattered light behind the structure was collected with an objective lens, whose position and, therefore, its focal point was moved step by step along the $z$ axis [Fig. 3(a)]. Hence, a set of $(x-y)$ cross sectional intensity distributions, i.e., a tomogram was acquired and subsequently averaged along the $y$ axis for visualization.

According to the measured intensity gain map, the device shows indeed a strong focusing effect with a definite focal point [Fig. 3(b)]. The integrated intensity value in the focal point at $Z=28 \mu \mathrm{m}$ exceeds that of the incident beam with a maximal gain value of $G=5.7$. The FWHM of the intensity peak along the $x$ axis in the focal point is $1.02 \mu \mathrm{m}$ which corresponds to a spot size conversion value of $\sim 18: 1$. By taking into the account the actual tapered nanopillar geometry a second 3D FDTD calculation was done showing the same focal distance [Fig. 3(c)]. Besides, both maps show similar patterns especially in the $Z>15 \mu \mathrm{m}$ and $|X|$ $>4 \mu \mathrm{m}$ region. Outside this regions, near to the scatterers the average intensity on the experimental map is significantly lower, which can be explained by the limited numerical aperture $(\mathrm{NA}=0.55)$ of the objective lens. The beams which point out from the $\theta=33^{\circ}$ half angle maximum cone were not collected by the lens.

icense or copyright, see http://apl.aip.org/apl/copyright.jsp 
(a)

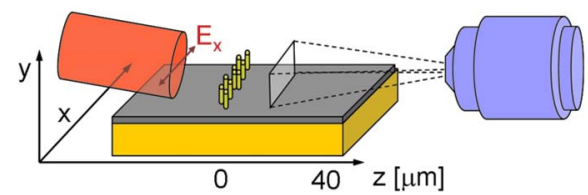

(b)



(c)

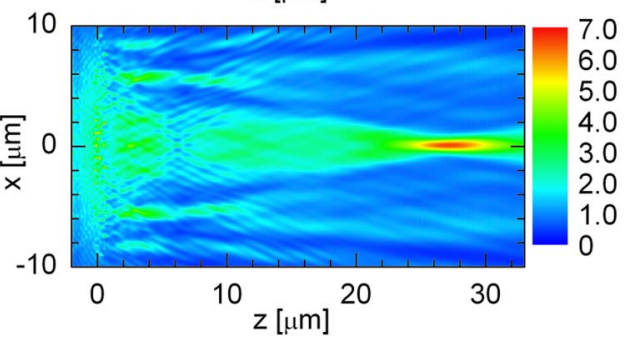

FIG. 3. (Color online) (a) Schematic illustration of the optical characterization setup. The TE polarized collimated laser beam grazing on the surface at an angle of $3.7^{\circ}$ is modulated by the nanopillar array. By moving the objective lens and hence its focal plane along the $z$ axis behind the specimen a series of the $x-y$ intensity distribution maps were recorded providing a complete 3D intensity distribution. (b) By taking the $y$-averaged $x-z$ intensity gain map, the desired focusing effect is obvious. (c) The theoretical intensity gain distribution calculated by 3D FDTD method shows very similar pattern features with the same focal distance of $Z=28 \mu \mathrm{m}$.

In conclusion, we have demonstrated that by using an engineered $\mathrm{ZnO}$ nanopillar array a very accurate manipulation of the light wave is feasible. In our experiment a focusing effect with a focal distance of $28 \mu \mathrm{m}$ was presented but according to our calculation by using a different scatterer arrangement a much shorter focal distance $(\sim 10 \mu \mathrm{m})$ can be easily reached. We believe that the $\mathrm{ZnO}$ nanopillar lens can be a promising tool for focusing the light coming from an external source into a specific volume on the chip, which can be either a rectangular or photonic waveguide as well as a $\mathrm{ZnO}$ nanowire based photodetector. However, we also expect that the real potential of the presented method will be utilized when the scatterers not only modulate but also emit the light enabling directionally emitting devices ${ }^{10}$ operating in the blue or near UV region.

This study was performed through Special Coordination Funds for Promoting Science and Technology from the MEXT, Japan. The authors are grateful to Y. Misawa, S. Hara, K. Tamura, A. Ohi, and T. Kasaya for professional help with sample preparation and optical data analysis.

${ }^{1}$ U. Ozgur, Ya. I. Alivov, C. Liu, A. Teke, M. A. Reshchikov, S. Dogan, V. Avrutin, S.-J. Cho, and H. Morkoc, J. Appl. Phys. 98, 041301 (2005).

${ }^{2}$ Z. L. Wang, J. Phys.: Condens. Matter 16, R829 (2004).

${ }^{3}$ R. Konenkamp, R. C. Word, and C. Schlegel, Appl. Phys. Lett. 85, 6004 (2004).

${ }^{4}$ M. H. Huang, S. Mao, H. Feick, H. Yan, Y. Wu, H. Kind, E. Weber, R. Russo, and P. Yang, Science 292, 1897 (2001).

${ }^{5}$ J. C. Johnson, H. Yan, R. D. Schaller, P. B. Petersen, P. Yang, and R. J. Saykally, Nano Lett. 2, 279 (2002).

${ }^{6}$ H. Kind, H. Yan, B. Messer, M. Law, and P. Yang, Adv. Mater. (Weinheim, Ger.) 14, 158 (2002).

${ }^{7}$ B. Xiang, P. Wang, X. Zhang, S. A. Dayeh, D. P. R. Aplin, C. Soci, D. Yu, and D. Wang, Nano Lett. 7, 323 (2007).

${ }^{8}$ A. Hakansson and J. Sanches-Dehesa, Appl. Phys. Lett. 87, 193506 (2005).

${ }^{9}$ L. Sanchis, A. Hakansson, D. Lopez-Zanon, J. Bravo-Abad, and J. Sanchez-Dehesa, Appl. Phys. Lett. 84, 4460 (2004).

${ }^{10}$ A. Hakansson, H. T. Miyazaki, and J. Sanches-Dehesa, Phys. Rev. Lett. 96, 153902 (2006).

${ }^{11}$ P. Selinger, M. Mahvash, Ch. Wang, and A. F. Levi, J. Appl. Phys. 100, 034310 (2006)

${ }^{12}$ A. Hakansson, F. Cervera, and J. Sanches-Dehesa, Appl. Phys. Lett. 86, 054102 (2005)

${ }^{13}$ Y.-J. Kim, Ch.-H. Lee, Y. J. Hong, and G.-Ch. Yi, Appl. Phys. Lett. 89, 163128 (2006).

${ }^{14}$ J. Cui and U. Gibson, Nanotechnology 18, 155302 (2007).

${ }^{15}$ M. Andrés Vergés, A. Mifsud, and C. J. Serna, J. Chem. Soc., Faraday Trans. 86, 959 (1990).

${ }^{16}$ T. Nagata, P. Ahmet, Y. Z. Yoo, K. Yamada, K. Tsutsui, Y. Wada, and T. Chikyow, Appl. Surf. Sci. 252, 2503 (2006).

${ }^{17}$ P. F. Titchmarsh, Proc. Phys. Soc. London 53, 391 (1941). 